\title{
Acetylcarnitine and Free Carnitine in Body Fluids before and after Birth
}

\author{
MILAN NOVAK, ${ }^{(4)}$ PAUL B. WIESER, MARIA BUCH, AND PETER HAHN \\ Department of Pediatrics, University of Miami School of Medicine (M. N., P. B. W., M. B.), Miami, Florida, USA, \\ and Centre for Developmental Medicine, University of British Columbia (P. H.), Vancouver, \\ British Columbia, Canada
}

\begin{abstract}
Summary
The acetylcarnitine concentration in amniotic fluid fell significantly between 33 and 37 and 40 weeks of gestation. It was significantly higher in samples that had a low lecithin to sphingomyelin ratio. The acetylcarnitine content of tracheal fluid was higher in samples obtained from premature newborns. The major excretory form of carnitine is the acetate ester and only in adults does the urine contain significant amounts of free carnitine. The level of acetylcarnitine in plasma significantly increases after the first day of life; there was no difference in the plasma levels of carnitine between full-term and premature newborns. Plasma-free carnitine and acetylcarnitine levels were significantly lower in infants who were receiving a soybean-based formula. A positive correlation was found between the plasma level of ketone bodies and that of acetylcarnitine.
\end{abstract}

\section{Speculation}

A characteristic of early postnatal adaptation in human newborns is a high dependence on the utilization of fatty acids derived from endogenous sources and increasingly from digested milk triglycerides. This essential energy-producing mechanism seems to be facilitated by carnitine, of which a considerable amount is derived from milk.

It is well known that the change from fetal life to extrauterine existence entails, among other things, changes in the supply and utilization of nutrients. In mammals, including humans, the "prenatal diet" can be considered as one high in carbohydrate, postnatally as a high-fat diet $(1,18)$. This change is accompanied by a rapid rise in blood levels of free fatty acids and ketone bodies after birth $(26,28,38)$. This rapid elevation of free fatty acid blood levels in human newborns during or shortly after birth is due to the release of fatty acids from adipose tissue mainly in response to $\beta$ adrenergic stimulation of lipolysis $(29,39)$. Later high levels of free fatty acid in blood reflect intestinal absorption of fatty acids from triglycerides supplied in the diet (18). Thus, $\beta$ oxidation of free fatty acid would seem to be a very important source of energy in the newborn. Carnitine ( $\gamma$-trimethylamino- $\beta$-hydroxybutyrate) is essential for optimum oxidation of fatty acids in mitochondria (2), since it facilitates transport of fatty acid across the mitochondrial membrane:

\section{Acetyl coenzyme A + Carnitine $\rightarrow$ acetylcarnitine + coenzyme A}

There is no doubt that carnitine and the carnitine transferases are essential for fatty acid oxidation after birth. We have reported relatively constant individual levels of free carnitine after birth in whole blood (35). However, there is substantial carnitine excretion in urine (35) before onset of feeding. Intake of exogenous carnitine varies with the type of milk fed. Formulas with a soybean base have no detectable carnitine (35).

The rapid postnatal increase in fat utilization in the rat is accompanied by a rise in hepatic $(2,3)$, cardiac $(42,43)$, brain (19), and brown fat (19) carnitine palmitoyl transferase and carnitine acetyl transferase activities. In man, carnitine palmitoyl transferase activity in white fat also rises rapidly after birth (27, $35)$.

In spite of the above changes, the level of free plasma carnitine changes only slightly in the perinatal period (35). However, mainly because of technical difficulties, the concentration of carnitine esters in body fluids has not been reported. Recently, we showed (16) that during starvation in man, blood and urine levels of acetylcarnitines increase considerably, while there is a concomitant decrease of free carnitine. We also noted (36) that in newborn rats, plasma levels of acetylcarnitine increased rapidly after birth and decreased only when the animals were weaned to a highcarbohydrate diet. Hence, it was decided to determine acetylcarnitine levels in plasma, urine, and amniotic fluid in human newborns.

Based on the postulation that in human newborns as in adults, the free carnitine concentration in plasma probably represents an equilibrium between intestinal absorption, biosynthetic activity of the liver, uptake and release by tissues, and renal clearance (15), we began to study the plasma levels of free and acetylcarnitine in babies receiving carnitine-free diets (soybean formulas). Such studies may help to determine whether exogenous carnitine is important in maintaining optimal carnitine blood levels and fatty acid oxidation after birth.

\section{MATERIALS AND METHODS}

Aliquots of amniotic fluid were obtained from samples used for diagnostic purposes. Samples of newborn tracheal fluid were obtained immediately after birth. Mixed cord blood was obtained immediately after delivery and capillary blood from newborns was obtained by heelprick using heparinized pipets. Written informed consent was obtained from the parents of the newborns before the sample was taken (47). The blood samples were centrifuged as soon as possible after collection, and the serum as well as the above mentioned fluids were stored at $-20^{\circ}$ or under liquid nitrogen until assayed. Small samples of urine $(1-2 \mathrm{ml})$ were obtained from newborns before food intake and after 4 days of life as well as from adult (nonpregnant) women for determination of the presence and concentration of free and bound carnitine.

Free carnitine was determined according to the method of McGarry and Foster (24), which is a modification of the original method of Cederblad and Lindstedt (12). Total carnitine was determined by alkaline hydrolysis (32) followed by carnitine assay as previously described (24). The difference between free and total carnitine was considered to be acetylcarnitine. To determine that this was indeed the case, acetylcarnitine was determined directly 
(31) in some samples. This direct assay could not be utilized for all of the samples because the method requires a large amount of sample which was not always available, especially in the case of the newborn plasma samples. However, in some samples that were large enough to be compared, good agreement was found between the two methods (Table 1).

$\beta$-Hydroxybutyric acid was determined according to the method of Persson (33). The lecithin to sphingomyelin ratio (L/S ratio) was determined in amniotic fluid samples obtained for evaluation of fetal lung maturity (17) according to the method of Borer et al. (7).

Gestational age of the newborns was estimated according to the criteria of Brazie and Lubchenco (8).

Carnitine acetyltransferase was supplied by Sigma Chemical Co. (44) and L-carnitine by Grand Island Biological Co. The other chemicals were of the highest purity obtainable.

\section{RESULTS}

\section{AMNIOTIC FLUID}

There was a significant $(P<0.001)$ drop in acetylcarnitine concentration between 33 and 37 and 40 weeks gestation (Fig. 1)

Table 1. Comparison of two methods for the determination of carnitine ${ }^{1}$

\begin{tabular}{ccc}
\hline & \multicolumn{2}{c}{ Acetylcarnitine (nmoles/ml) } \\
\cline { 2 - 3 } Sample & Method A & Method B \\
\hline Amniotic fluid & 3.0 & 2.7 \\
& 1.0 & 0.7 \\
Urine & 1.8 & 1.7 \\
& 15.0 & 13.9 \\
& 30.0 & 25.1 \\
& 95.0 & 102.0 \\
\hline
\end{tabular}

\footnotetext{
${ }^{1}$ Samples of amniotic fluid and urine were assayed directly for acetylcarnitine according to the spectrophotometric method of Pearson et al. (31) (method A). The acetylcarnitine present in an additional aliquot was measured according to the method of McGarry and Foster (24) (method B).
}

\section{ACETYL CARNHTHE iN AMNIOTIC fLUD}

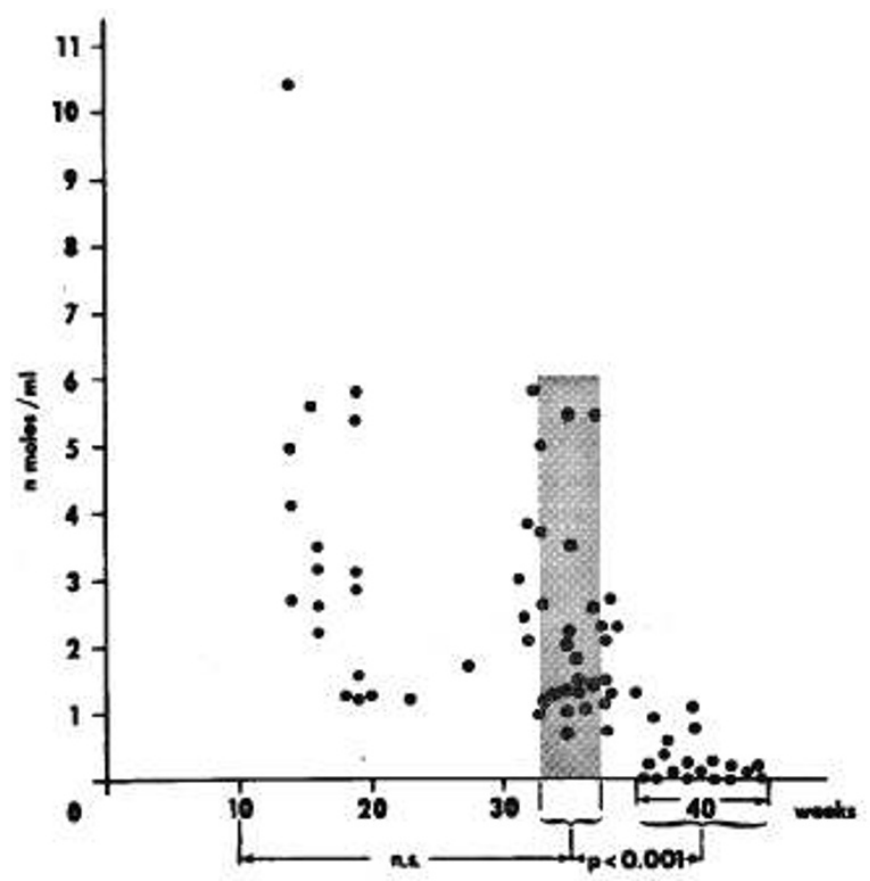

Fig. 1. Acetylcarnitine levels in amniotic fluid. The shaded area (33-37 weeks of gestation) is compared with full-term pregnancies.

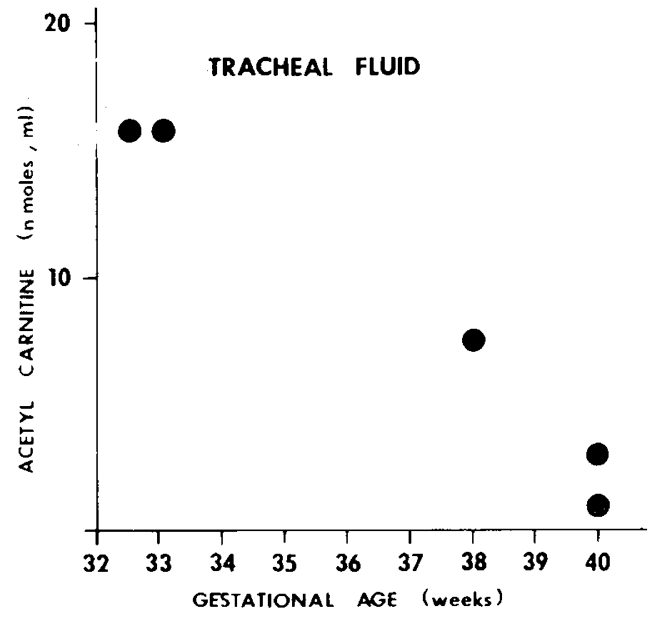

Fig. 2. Acetylcarnitine levels in tracheal fluid. Tracheal aspirates were obtained immediately after birth and the gestational age of the newborn was determined as indicated.

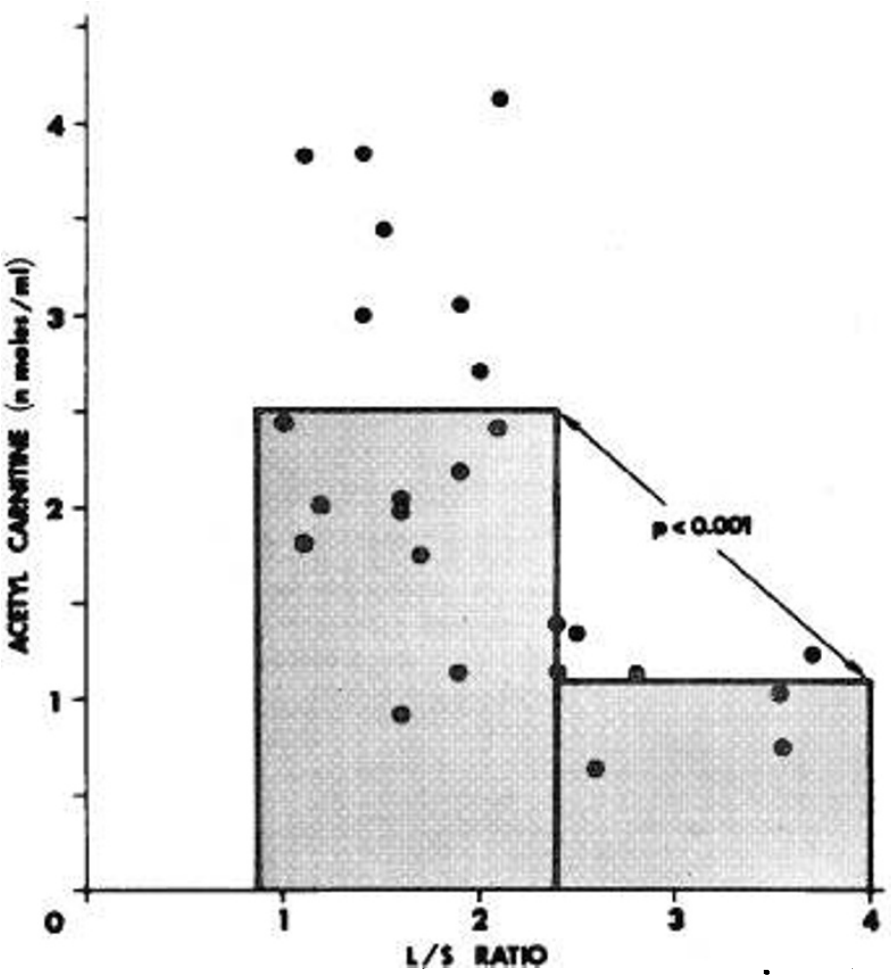

Fig. 3. Acetyicarnitine levels in amniotic fluid and the $\dot{L} / S$ ratio. Acetylcarnitine levels were determined in aliquots of amniotic fluid, which had been obtained for determination of the $\mathrm{L} / \mathrm{S}$ ratio.

which parallels the fall seen for free carnitine (21). At all ages, acetylcarnitine represents about $10 \%$ of the total amniotic fluid content of carnitine. The acetylcarnitine concentration in tracheal fluid was also higher in samples obtained from premature newborns suctioned immediately after birth (Fig. 2). This suggested that amniotic fluid acetylcarnitine may in part be derived from the trachea. Figure 3 shows that the acetylcarnitine concentration in amniotic fluid was significantly lower in samples that had a high $\mathrm{L} / \mathrm{S}$ ratio, i.e., as the lungs mature they produce less acetylcarnitine and more surfactant.

\section{URINE}

The urinary levels of free and acetylcarnitine are shown in Table 2. In infants, the major excretory form of carnitine is the 
acetate ester. The acetylcarnitine content per milliliter is the same in all age groups. Free carnitine content, however, is significantly higher in adults.

\section{BLOOD PLASMA}

The concentration of free carnitine and acetylcarnitine in cord blood and capillary blood plasma of premature and normal infants is given in Figures 4 and 5. The free carnitine levels are subject to wide individual variation (see also 19), but the level of acetylcarnitine increases significantly after the first day of life $(P<0.005)$ and, as with free carnitine, there was no difference between fullterm and premature plasma levels.

The effect of diet with and without carnitine on plasma-free and acetylcarnitine levels is shown in Figures 6 and 7. In the case of both premature and full-term infants, plasma carnitine levels were significantly lower in infants who were receiving a soybeanbased formula (Fig. 6) and the same was true for acetylcarnitine (Fig. 7). However, from the published composition of breast milk and various commercial formulas given in Table 3 , it is clear that amino acid precursors of carnitine, lysine, and methionine are present at higher concentrations in soybean-based formulas than in human milk.

Figure 8 is a comparison of the levels of plasma $\beta$-hydroxybutyrate and acetylcarnitine as percent of total carnitine. There is a positive correlation between the ketone body levels and acetylcarnitine $(y=30.6 \times-954.3, r=0.91)$.

Table 2. Concentration of free carnitine and acetylcarnitine in the urine of newborns and adult (nonpregnant) women ${ }^{1}$

\begin{tabular}{lll}
\hline \multicolumn{1}{c}{ Age group } & $\begin{array}{c}\text { Free carnitine } \\
(\mathrm{nmoles} / \mathrm{ml})\end{array}$ & $\begin{array}{c}\text { Acetylcarnitine } \\
\text { (nmoles } / \mathrm{ml})\end{array}$ \\
\hline $\begin{array}{l}\text { Newborns before first } \\
\text { feeding }\end{array}$ & $6.96 \pm 0.75$ & $31.71 \pm 7.29^{2}(n=12)$ \\
$\begin{array}{l}4 \text { days old } \\
\text { Adults }\end{array}$ & $9.62 \pm 3.62$ & $27.60 \pm 10.43^{3}(n=6)$ \\
\hline
\end{tabular}

${ }^{1}$ Statistical evaluation by Student's $t$ test as indicated.

${ }^{2} P<0.005$ compared with free carnitine.

${ }^{3} P<0.05$ compared with free carnitine.

${ }^{4} P<0.005$ compared with newborns.

${ }^{5} P<0.05$ compared with 4-day olds.

${ }^{6} P$ not significant compared with free carnitine.

\section{DISCUSSION}

Carnitine content of tissues as well as of body fluids has often been reported in terms of free carnitine. This is particularly true in the case of blood $(6,9-11,37)$ and urine (11). As a result of improvements in methodology, it is possible to extend these measurements to include acetylcarnitine, which can now be measured in very small volumes $(12,24,30)$.

Although the function and significance of acetylcarnitine and the enzyme catalyzing its production, acetyl CoA: carnitine-oacetyltransferase (EC 2.3.1.7), acetyl CoA, is not exactly known, the increase of acetylcarnitine in blood plasma most probably reflects the accumulation of acetylcarnitine in tissues in response to the elevated rate of generation of acetyl CoA, presumably by fatty acid oxidation, since it has been shown that an elevation in acetyl CoA production in mitochondria is reflected by increased
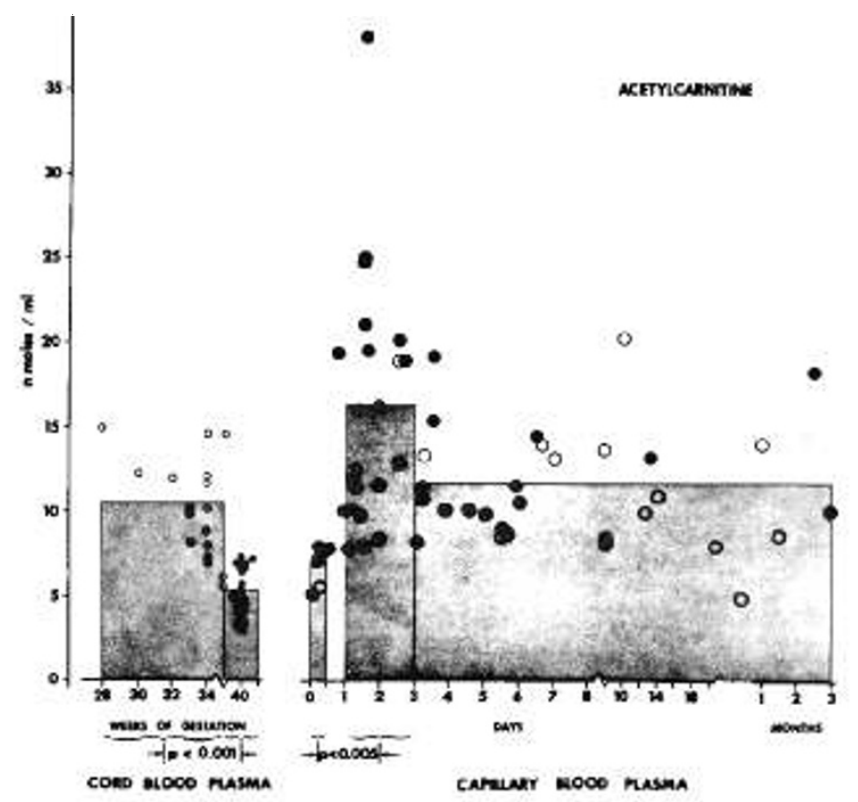

Fig. 5. Plasma levels of acetylcarnitine in umbilical cord and newborn capillary blood. Acetylcarnitine was determined in cord and newborn capillary blood in premature $(O)$ and normal full-term infants $(\Theta)$.

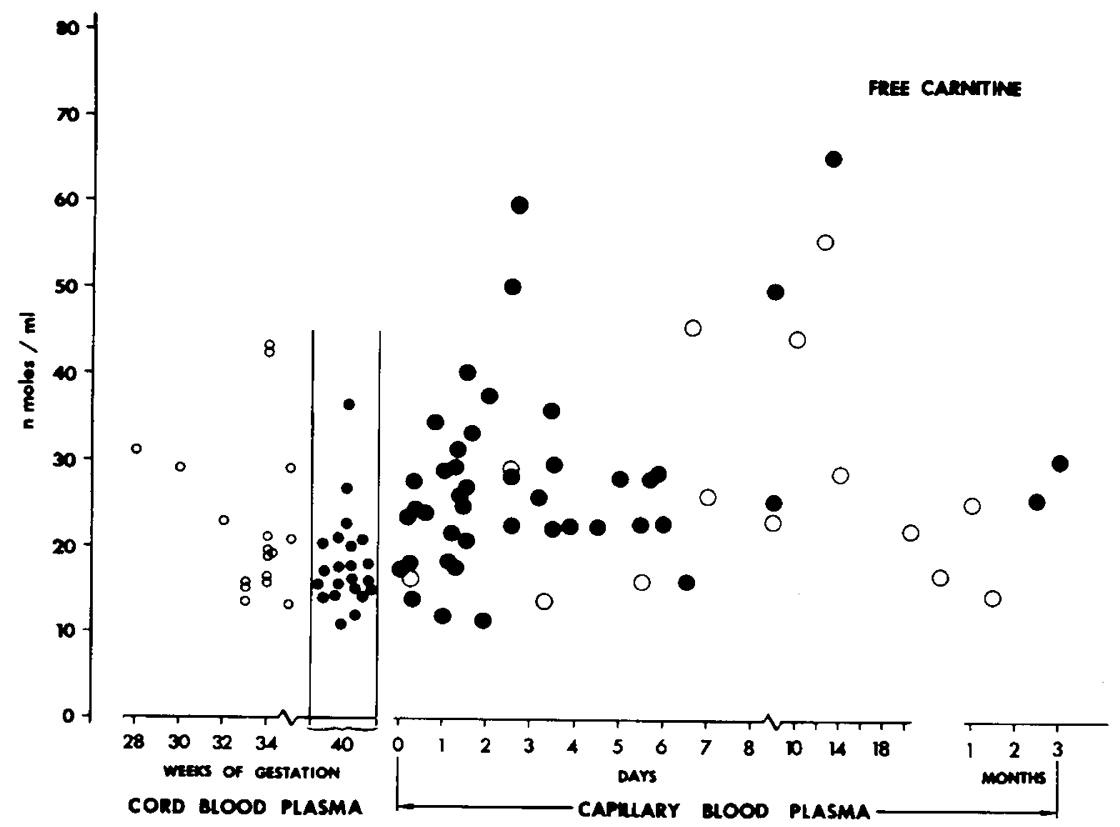

Fig. 4. Plasma levels of free carnitine in umbilical cord and newborn blood. Free carnitine was determined in cord and newborn capillary blood in premature $(O)$ and normal full-term infants $(\bullet)$. 


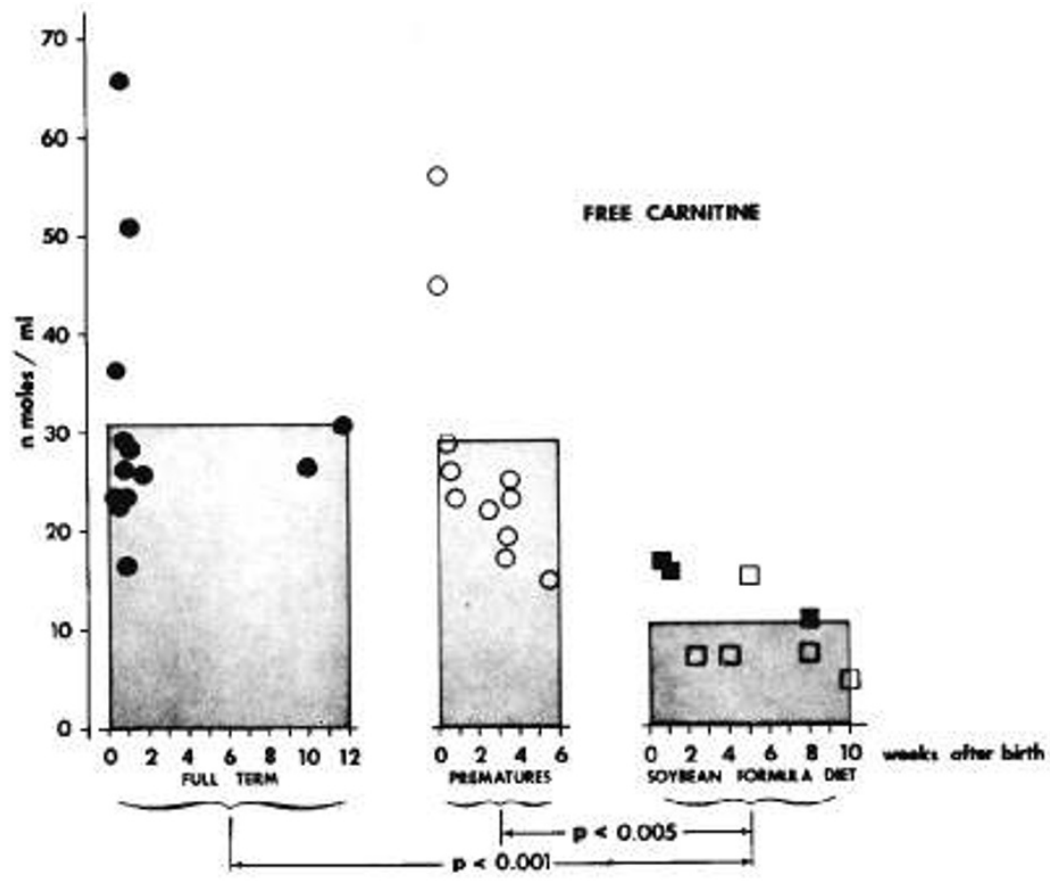

Fig. 6. Plasma levels of free carnitine in infants fed various diets. Free carnitine was determined in capillary blood from normal ( $)$ and premature F) infants maintained on either breast milk or a cow's milk-based formula. The free carnitine levels in the third column are from normal ( $\square$ ) and premature $(\square)$ infants maintained on soybean-based formulas.

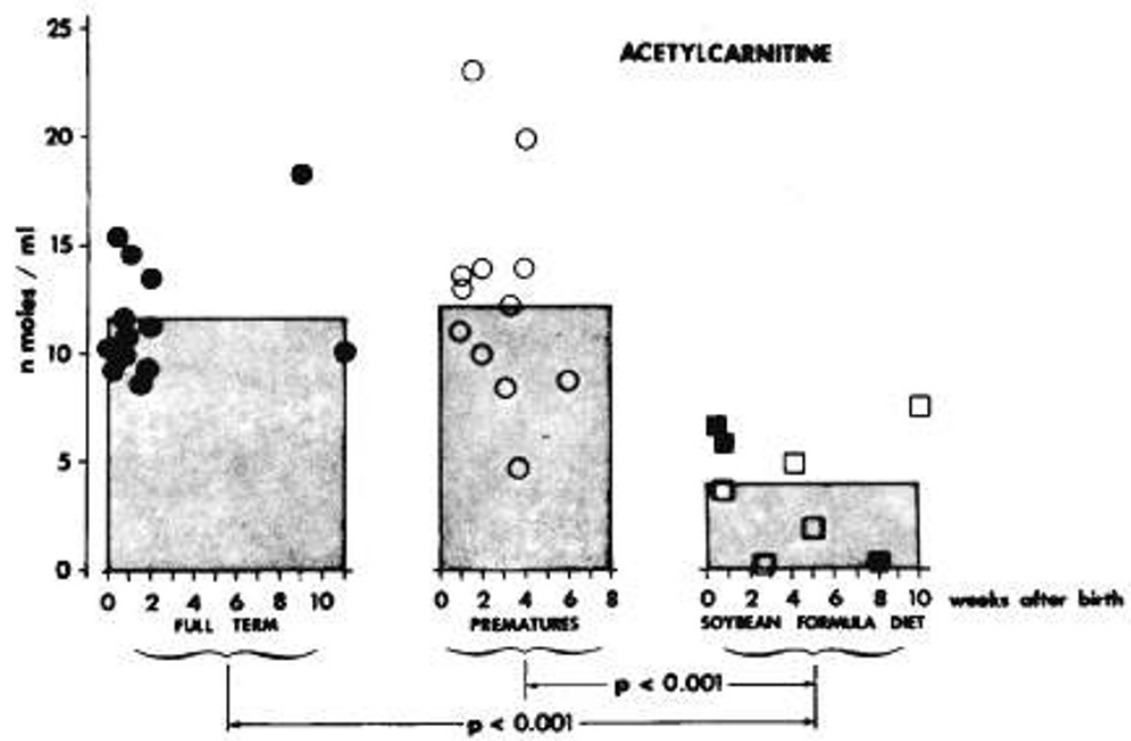

Fig. 7. Plasma levels of acetylcarnitine in infants fed various diets. Acetylcarnitine was measured in plasma from the same group of infants given in Figure 6.

Table 3. Content of methionine and lysine in human breast milk, some cows' milk-based formulas, and soybean-based formulas in $m g /$ liter

\begin{tabular}{|c|c|c|c|c|}
\hline & Lysine & Methionine & Reference & $\begin{array}{c}\text { Total carnitine } \\
\text { (nmoles/ml) }\end{array}$ \\
\hline$\underset{\text { milk }}{\operatorname{Human}}$ breast & 730 & 250 & $(14,22,23)$ & $39-63(35)$ \\
\hline Similac & 1210 & 430 & $(14,22,23)$ & $60-90(35)$ \\
\hline Enfamil & 1230 & 390 & $(14,22,23)$ & $50-80(35)$ \\
\hline Prosobee 20 & 1360 & 470 & $(14,22,23)$ & $0(35)$ \\
\hline Isomil 20 & 1180 & 330 & $(14,22,23)$ & $0(35)$ \\
\hline Nursoy & $-{ }^{1}$ & - & & $0(35)$ \\
\hline
\end{tabular}

${ }^{1}$ Data not available. tissue levels of acetylcarnitine $(4-6,15,40)$. The rapid increase in acetylcarnitine levels in plasma and relatively low and constant levels of free plasma carnitine in newborns (Figs. 4 and 5) are in agreement with this concept. However, cord blood plasma levels of acetylcarnitine taken during premature delivery are also elevated (Fig. 5), while the free carnitine plasma levels do not differ (Fig. 4) from "normal" values. There is general agreement that fatty acid oxidation is of minor importance during the fetal period $(1,41)$. Nevertheless, fatty acid plasma levels are very high very soon after birth (28) and it is likely that labor triggers fatty acid release, thus causing elevated acetylcarnitine levels.

As indicated by the concomitant decrease in acetylcarnitine concentration in both amniotic (Fig. 1) and tracheal fluids (Fig. 2) and by the relatively high concentration of acetylcarnitine 

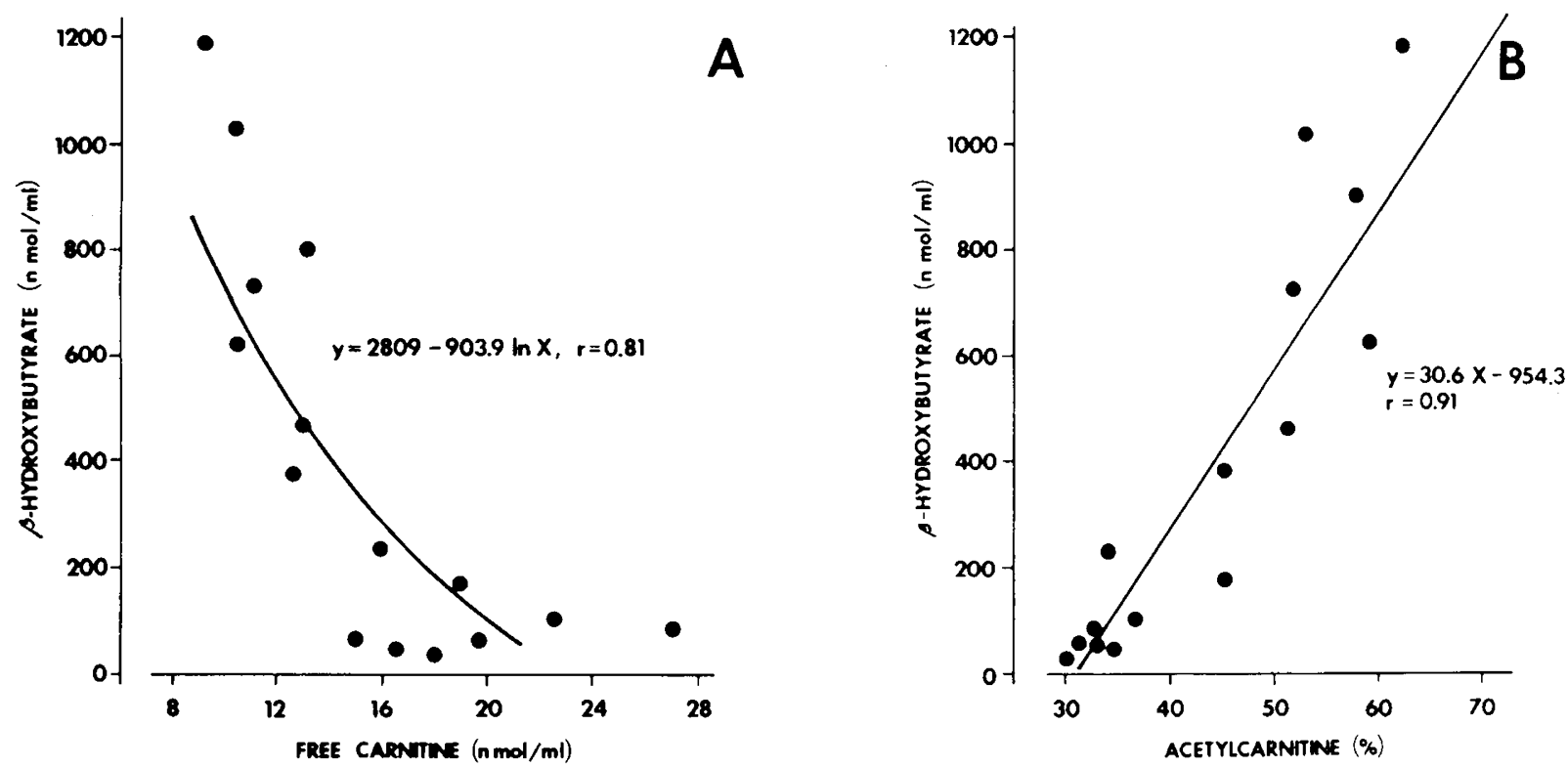

Fig. 8. A, Negative logarithmic correlation between free carnitine and $\beta$-hydroxybutyrate plasma levels in newborns $48 \mathrm{hr} \pm 4 \mathrm{hr}$ after birth. B, Linear correlation of percent of acetylcarnitine of total carnitine content with $\beta$-hydroxybutyrate.

in tracheal fluid, fetal lung may also be a source of amniotic fluid acetylcarnitine in addition to fetal urine. It is also tempting to speculate that the negative correlation between acetylcarnitine and the $\mathrm{L} / \mathrm{S}$ ratio reflects changes in consumption of acetyl $\mathrm{CoA}$ for the biosynthesis of palmitate. On the other hand, the decreased levels of free carnitine in maternal blood toward the end of gestation (21) leading to a decreased supply of free carnitine to the fetus may be an additional factor, causing low carnitine and acetylcarnitine levels in amniotic fluid.

The significance of dietary carnitine for the human newborn is unknown, but some reports indicating the importance of carnitine in newborn mammals have appeared $(3,20,34)$. One of the objectives of this study is to examine the possibility that the dietary carnitine the human newborn receives may possibly be required for the optimal capability to oxidize fatty acids. This postulation is supported by our data showing a decreased level of free carnitine and acetylcarnitine in infants receiving carnitine-free diets (Figs. 6 and 7) and by some earlier published data. We wish to emphasize several points from these studies:

1. In neonatal rats, carnitine ingested from milk is rapidly accumulated in tissues (20), and the increase of ketogenic capacity in the neonatal rat is related to carnitine accumulation in liver $(25,34)$.

2. Previous studies from this laboratory have suggested that carnitine is also accumulated by human newborn tissues as indicated by increases in free and long chain acetylcarnitine in white subcutaneous adipose tissue (35).

3. Carnitine is excreted in urine before the onset of feeding (35), and we have determined that acetylcarnitine is the main form in which carnitine is excreted (Table 2).

In addition to the influence of free fatty acid availability and glucagon- and insulin-mediated alterations in the rate of ketogenesis, carnitine acetyltransferase activity in liver has also been shown to be of importance in the production of ketone bodies (13). In human newborns as well as in other mammalian neonates, increased fatty acid oxidation leads to a marked increase in acetyl CoA production which exceeds the capacity for its oxidation via the Krebs cycle. Condensation of acetyl CoA molecules to form ketone bodies and a simultaneous elevated acetylation of carnitine is reflected by a positive correlation between $\beta$-hydroxybutyrate and acetylcarnitine concentrations in plasma (Fig. 8).

To sum up, we feel that since, during the neonatal period, fatty acid oxidation is the main pathway by which energy is produced and the dietary intake of carnitine may be more important than prenatally and in the adult. It remains to be determined whether decreased plasma levels of free and esterified carnitine in infants fed carnitine-free diets has a detrimental effect on the capacity of tissues to metabolize fatty acids and to produce the necessary energy for optimal growth and development.

\section{REFERENCES AND NOTES}

1. Adams, P. A. J.: Control of glucose metabolism in the human fetus and newborn infant. In: R. Levine and R. Luft: Advances in Metabolic Disorders, Vol. 5 (Academic Press, NY, 1971).

2. Augenfeld, J., and Fritz. I. B.: Carnitine palmitoyltransferase activity and fatty acid oxidation by livers from fetal and neonatal rats. Can. J. Biochem.. 48: 288 (1970).

3. Bieber, L. L., Markwell. M. A.. Blair, M.. and Helmrath. T. A.: Studies on the development of carnitine palmitoyltransferase and fatty acid oxidation in liver mitochondria of neonatal pigs. Biochim. Biophys. Acta. 326: 145 (1973).

4. Bohmer, T.: Tissue concentrations of activated fatty acids (acylcarnitines) and the regulation of fatty acid metabolism. Biochim. Biophys. Acta. 144: 259 (1967).

5. Bohmer, T., Normu, K., and Bremer. J.: The relative amounts of long chain acylcarnitine, acetylcarnitine and free carnitine in organs of rats of different nutritional states and with alloxan diabetes. Biochim. Biophys. Acta. 125: 244 (1966).

6. Bohmer, T., Rydning, A., and Solberg, H.: Carnitine levels in human serum in health and disease. Clin. Chim. Acta. 57: 55 (1964).

7. Borer, Jr., R. C., Gluck, L.. Freeman, R. K., and Kulovich. M. V.: Prenatal prediction of the respiratory distress syndrome (RDS). Pediatr. Res.. 5: 655 (1971).

8. Brazie, J. W., and Lubchenco, L. O.: The estimation of gestational age chart. In: C. H. Kempe, H. K. Silver, and D. O'Brien: Current Pediatric Diagnosis and Treatment, 3rd ed., ch. 3 (Lange Medical Publications. Los Altos. CA. 1974). Los Altos, CA. 1974).

9. Brooks, E. E., and McIntosh. J. E.: Turnover of carnitine by rat tissue. Biochem. J., 148: 439 (1975)

10. Cederblad, G.: Plasma carnitine and body composition. Clin. Chim. Acta, 67: 207 (1976).

11. Cederblad, G., and Lindstedt, S.: Excretion of L-carnitine in man. Clin. Chim. Acta, 33: 117 (1971).

12. Cederblad, G., and Lindstedt, S.: A method for the determination of carnitine in the picomole range. Clin. Chim. Acta, 37: 235 (1972).

13. Felig, P.: Recent developments in body fuel metabolism. In: N. Freinkel: The Year in Metabolism, p. 116, 124, 126 (Plenum Medical Book Co.. NY. 1977).

14. Fomon, S. J.: Infant Nutrition, 2nd ed. (W. B. Saunders Co.. Philadelphia. 1974).

15. Fritz, 1. B.: Carnitine and its role in fatty acid metabolism. Adv. Lipid Res.. 1: 285 (1963).

16. Frohlich, J., Seccombe, D. W., Hahn. P.. Dodek, P.. and Hynie. I.: The effect of fasting on free and esterified carnitine levels in human serum/urine: Correlation with serum levels of FFA and $\beta$-hydroxybutyrate. Metabolism. 27: 555 (1978).

17. Gluck, L., and Kulovich, M.: Fetal lung maturity. In: F. Adamsons and H. A. Fox: Progress in Clinical and Biological Research. Preventability of Perinatal Injury, Vol. 2, p. 135 (Alan R. Liss Inc.. NY. 1975).

18. Hahn, P., and Koldovsky, O.: Utilization of nutrients during postnatal develop- 
ment (Pergamon Press, Oxford, 1966).

19. Hahn, P., and Skala, J.: Carnitine and brown adipose tissue metabolism in the rat during development. Biochem. J., 127: 107 (1972).

20. Hahn, P., and Skala, J.: The role of carnitine in brown adipose tissue of suckling rats. Comp. Physiol. Biochem., SIB: 507 (1975).

21. Hahn, P., Skala, J. P., Seccombe, D. W., Frohlich, J., Penn-Walker, D., Novak, M., Hynie, D., and Towell, M. E.: Carnitine content of amnioic fluid and maternal and umbilical blood. Pediatr. Res., 11: 878 (1977).

22. Macy, I. G.: The composition of milks, p. 63 (National Academy of SciencesNational Research Council, Washington, 1953).

23. Macy, I. G., and Kelly, H. J.: Human milk and cows milk in infant nutrition. In: S. K. Kon and A. T. Cowie: Milk: The Mammary Gland and Its Secretion, Vol. 2, p. 268 (Academic Press, NY, 1961).

24. McGarry, J. D., and Foster, D. W.: An improved and simplified radioisotopic assay for the determination of free and esterified carnitine. J. Lipid Res., 17: 277 (1976).

25. McGarry, J. D., Robles-Valdes, C., Foster, D. W.: Role of carnitine in hepatic ketogenesis. Proc. Natl. Acad. Sci. USA, 72: 4385 (1975).

26. Melichar, V., Drahota, Z., and Hahn, P.: Changes in the blood levels of acetoacetate and ketone bodies in newborn infants. Biol. Neonate, 8: 348 (1965).

27. Novak, M., Hahn, P., Penn, D., Monkus, E., and Skala, J.: The role of carnitine in subcutaneous white adipose tissue from newborn infants. Biol. Neonate, 23 : 11 (1973).

28. Novak, M., Melichar, V., Hahn, P., and Koldovsky, O.: Levels of lipids in the blood of newborn infants and effect of glucose administration. Physiol. Bohemoslov., 10: 488 (1961).

29. Novak, M., and Monkus, E.: Metabolism of subcutaneous adipose tissue in the immediate postnatal period of human newborns. I. Developmental changes in lipolysis and glycogen content. Pediatr. Res., 6: 73 (1972).

30. Parvin, R., and Pande, S. V.: Microdetermination of (-) carnitine and carnitine acetyltransferase activity. Anal. Biochem., 79: 190 (1977).

31. Pearson, D. J., Chase, J. F. A., and Tubbs, P. K.: Methods Enzymol., 14: 621 (1968).

32. Pearson, D. J., and Tubbs, P. K.: Tissue levels of acid insoluble carnitine in rat heart. Biochim. Biophys. Acta, 84: 772 (1964).

33. Persson, B.: Determination of plasma acetoacetate and $D-\beta$-hydroxy-butyrate in newborn infants by an enzymatic fluorometric method. Scand. J. Clin. Invest.,
25: 9 (1970).

34. Robles-Valdes, C., McGarry, J. D., and Foster, D. W.: Maternal-fetal carnitine relationship and neonatal ketosis in the rat. J. Biol. Chem., 251: 6007 (1976).

35. Schmidt-Sommerfeld, E., Novak, M., Penn, D., Wieser, P., Buch, M., and Hahn, P.: Carnitine and development of newborn adipose tissue. Pediatr. Res. (in press).

36. Seccombe, D. W., Hahn, P., and Novak, M.: The effect of diet and development on blood levels of free and esterified carnitine in the rat. Biochim. Biophys. Acta, 528: 483 (1978).

37. Snoswell, A. M., and Henderson, G. D.: Aspects of carnitine ester metabolism in sheep liver. Biochem. J., 119: 59 (1970).

38. Van Duyne, C. M., and Havel, R. J.: Plasma UFA concentration in fetal and neonatal life. Proc. Soc. Exp. Biol., 102: 299 (1959).

39. Van Duyne, C. M., Parker, H. L., Havel, R. J., and Holm, L. W.: Free fatty acid metabolism in fetal and newborn sheep. Am. J. Physiol., 199: 987 (1960).

40. Van Tol, A.: The effect of fasting on the acylation of carnitine and glycerophosphate in rat liver subcellular fractions. Biochim. Biophys. Acta, 357: 14 (1974).

41. Vernon, R. G., and Walker, D. G.: Changes in some enzymes involved in glucose utilization and formation in developing rat. Biochem. J., 106: 321 (1968).

42. Warshaw, J. B., and Ferry, M. L.: Cellular energy metabolism during fetal development. J. Cell Biol., 44: 354 (1970).

43. Witteis, B., and Bressler, R.: Lipid metabolism in the newborn heart, J. Clin. Invest., 44: 1639 (1965).

44. Supplied by Sigma Chemical Co., St. Louis, Mo. as were all other chemicals except where noted.

45. The authors are indebted to Jeanne Cesteros for her assistance in the preparation of this manuscript and to Marcia Sills and Tim Brooks for their technical assistance.

46. This research was supported by NIH Grant HD04946.

47. This research project was reviewed and approved by the Humai Research Committee of the University of Miami School of Medicine. The reason for collecting the sample was always explained to the mothers, and the sample was collected only after obtaining a written informed consent.

48. Requests for reprints should be addressed to: Dr. Milan Novak, Mailman Center for Child Development, P. O. Box 520006, Biscayne Annex, Miami, FL 33152. 49. Received for publication November 14, 1977.

50. Accepted for publication February 24, 1978. 